\title{
Influence of Physical Exercise on Oxidative Stress and Inflammation in Hemodialysis Patients
}

\author{
Marta Esgalhado ${ }^{1^{*}}$ and Denise Mafra ${ }^{2}$ \\ ${ }^{1}$ University of Medicine of Lisbon, Post-Graduation in Clinical Nutrition, Lisbon, Portugal \\ ${ }^{2}$ Fluminense Federal University, Post-Graduation in Cardiovascular Sciences, Niterói-RJ, Brazil
}

"Corresponding author: Marta Esgalhado, University of Medicine of Lisbon, Post-Graduation in Clinical Nutrition, Rua Agostinho Neto, n²2 $5^{\circ} \mathrm{G}, 1750-006$ Lisbon, Portugal, Tel: 351915660217; E-mail: martaesgalhado@hotmail.com

Rec date: Apr 28, 2015; Acc date: Apr 29, 2015; Pub date: May 02, 2015

Copyright: (c) 2015 Esgalhado M, et al. This is an open-access article distributed under the terms of the Creative Commons Attribution License, which permits unrestricted use, distribution, and reproduction in any medium, provided the original author and source are credited.

\section{Editorial}

Chronic kidney disease (CKD) is associated with oxidative stress and systemic inflammation, which promote cardiovascular disease (CVD), the leading cause of morbidity and mortality in this population, mainly in hemodialysis (HD) patients [1].

Mechanisms involved in the inactivation of the oxidative stress and inflammation have been stressed for being considered promising approaches to minimize cardiovascular complications. Among several strategies that are being studied to reduce these complications, physical exercise (PE) could represent a new non-pharmacological approach [2]. In fact, Koufaki et al. [3] demonstrated that 'some' physical activity compared to 'none', leads to cardiovascular mortality risk reduction of up to $50 \%$. Furthermore, CKD patients who performed exercises 2-5 times weekly have lower mortality compared to those practicing only once a week [4]. A recent study showed that nondialysis CKD patients had $26 \%$ higher risk for death when there was a reduction on $0.1 \mathrm{~m} / \mathrm{s}$ in gait speed test [5]. In a cohort of 2837 dialysis patients, the mortality of those patients who declared to practice $\mathrm{PE}$ was $5 \%$ compared to $11 \%$ in sedentary patients [6], Accordingly, epidemiological reports show that physical inactivity is an independent risk factor for development and progression of the rate of loss of kidney and cardiovascular function [3].

Recognizing this situation, several guidelines, including those of the Kidney Disease: Improving Global Outcomes (KDIGO) CKD clinical practice guideline [7], the National Kidney Foundation Kidney Disease Outcome Quality Initiative (K/DOQI) clinical practice guidelines [8], and the American College of Sports Medicine [9], recommend that patients with CKD practices regular PE, aiming for at least 30 minutes 5 times/week [7]. Despite being increasingly highlighted, PE is not yet routinely recommended by renal multidisciplinary care teams, regardless of meriting early intervention for optimum disease prevention and management [3]. In an attempt to improve PE adoption and adherence, a range of exercise programs have been developed for CKD patients, such as aerobic exercise, resistance exercise, and combined aerobic and resistance exercise; moreover several investigators have prescribed exercise training during routine HD treatment (intradialytic PE) [10-14].

A notable quantity of research has been conducted on PE in CKD patients, being the aerobic exercise the most studied, such as cycle ergometer or bicycle training [2]. These exercise programs vary according, intensity, duration and the use of different exercise frequencies; however, all of them have stressed the importance of a consistent practice of training between two or three times a week with moderate or vigorous intensity for 30 minutes or more [10]. The resistance exercise programs consist in progressive resistance training of upper extremity strengthening with free-weight dumbbells, lower extremity strengthening with weighted ankle cuffs, or use of the Thera-band stretch strap and other specific progressive resistance training for shoulders, hip, and abdominal musculature [10].

Regular PE seems to improve physical capacity, muscle strength, cardiovascular function, and health-related quality of life, including benefits on depressive symptoms, which may reduce mortality and hospitalization in CKD patients $[10,11,15]$.

According to Seong [10] no studies in CKD patients have been carried out to establish the impact of exercise on cardiovascular outcomes, even so, a few studies have examined the effects of PE on potential mediators of cardiovascular risk, such as oxidative stress. This mediator results from an imbalance between reactive oxygen species production and insufficient endogenous antioxidant defense mechanisms. As follows, oxidative stress promotes the activation of factors that induce the inflammatory processes, establishing a vicious cycle. This mechanism is directly related to the increased risk of developing CVD in CKD patients [2].

Although chronic PE can improve oxidative stress and inflammation in HD patients [12-14], acute PE seems to exacerbate them $[2,16]$.

Gordon et al. [12] described that oxidative stress markers decreased after 4 months of Hatha yoga training in HD patients. Wilund et al. [13] examined the effects of 4 months of intradialytic exercise training (cycling) and also observed that this exercise may improve CVD risk by decreasing novel risk factors including oxidative stress markers. Furthermore, previous results from our research group [14] showed that chronic resistance exercise (during 6 months) exerted antiinflammatory effects in HD patients.

In a recent study our research group assessed the effect of acute intradialytic strength $\mathrm{PE}$ and demonstrated that acute exercise was unable to reduce oxidative stress and inflammation, and in addition, it seems that reduced plasma SOD levels may exacerbate oxidative stress in these patients [2]. A previous study conducted by Fatouros et al. [16] examined the effects of acute aerobic PE and also observed an increase of oxidative stress markers in HD patients, probably due to diminished antioxidant defense.

Overall, is PE beneficial to patients with CKD, mainly in HD? The answer is "yes". Regular PE leads to increased physical capacity, muscle strength, cardiovascular function, improved health-related quality of life, and decreased morbidity and mortality without decreasing renal function in this population. Aerobic PE is the most common form of training in CKD patients and seems to be effective in 
improving CKD complications; however, resistance exercise may also be an alternative. Not only is the nature of exercise important, but also the duration of intervention and the frequency and intensity of sessions are crucial points to be discussed

\section{References}

1. Vaziri ND (2014) Gut microbial translocation in the pathogenesis of systemic inflammation in patients with end-stage renal disease. Dig Dis Sci 59: 2020-2022.

2. Esgalhado M, Stockler-Pinto MB, Ludmila FM, Cardozo CC, Barboza JE, et al. (2015) Effect of acute intradialytic strength physical exercise on the oxidative stress and inflammatory responses in HD patients. Kidney Res Clin Pract 34: 35-40.

3. Koufaki P, Greenwood S, Painter P, Mercer T (2015) The BASES expert statement on exercise therapy for people with chronic kidney disease. J Sports Sci.

4. Stack AG, Molony DA, Rives T, Tyson J, Murthy BV (2005) Association of physical activity with mortality in the US dialysis population. Am J Kidney Dis 45: 690-701.

5. Roshanravan B, Robinson-Cohen C, Patel KV, Ayers E, Littman AJ, et al. (2013) Association between physical performance and all-cause mortality in CKD. J Am Soc Nephrol 24: 822-830.

6. O'Hare AM, Tawney K, Bacchetti P, Johansen KL (2003) Decreased survival among sedentary patients undergoing dialysis: Results from the dialysis morbidity and mortality study wave 2 . Am J Kidney Dis 41: 447454 .
7. KDIGO 201 (2013) Clinical practice guideline for the evaluation and management of chronic kidney disease. J Int Soc Nephrol 3: 1-163.

8. K/DOQI Workgroup (2005) K/DOQI clinical practice guidelines for cardiovascular disease in dialysis patients. Am J Kidney Dis 45: S1-153.

9. American College of Sports Medicine (2013) ACMS's Guidelines for Exercise Testing and Prescription Medicine. (9thedn), Lippincott Williams \& Wilkins/Wolters Kluwer Health. Philadelphia, USA.

10. Seong EY (2015) Acute intradialytic exercise and oxidative stress in hemodialysis patients. Kidney Res Clin Pract 34:1-3.

11. Heiwe S, Jacobson SH (2014) Exercise training in adults with CKD: a systematic review and meta-analysis. Am J Kidney Dis 64: 383-393.

12. Gordon L, McGrowder DA, Pena YT, Cabrera E, Lawrence-Wright MB (2013) Effect of yoga exercise therapy on oxidative stress indicators with end-stage renal disease on hemodialysis. Int J Yoga 6: 31-38.

13. Wilund KR, Tomayko EJ, Wu PT, Ryong Chung H, Vallurupalli S, et al. (2010) Intradialytic exercise training reduces oxidative stress and epicardial fat: a pilot study. Nephrol Dial Transplant 25: 2695-2701.

14. Moraes C, Stockler-Pinto MB, Lobo JC, Barros AF, Wady MB, et al. (2012) Resistance Exercise Program: Intervention To Reduce Inflammation And Improve Nutritional Status In Hemodialysis Patients. Kidney Res Clin Pract 31: A58.

15. Mafra D, Fouque D (2014) Lower physical activity and depression are associated with hospitalization and shorter survival in CKD. Clin J Am Soc Nephrol 9: 1669-1670.

16. Fatouros IG, Pasadakis P, Sovatzidis A, Chatzinikolaou A, Panagoutsos S, et al. (2008) Acute exercise may exacerbate oxidative stress response in hemodialysis patients. Nephron Clin Pract 109: c55-64. 